\title{
Prevalence De La Maladie Du Deperissement De L'ananas (Wilt) Et Perception Des Producteurs Au Benin
}

\section{Houndédji C. D.}

Laboratoire de Diagnostic et de Soutien à la Protection des Végétaux,

Direction de la Production Végétale (DPV), Bénin, Porto-Novo Département de Biologie Végétale, Faculté des Sciences et Techniques, Université d’Abomey-Calavi, Bénin, Abomey-calavi

\section{Zandjanakou-Tachin $M$.}

Laboratoire de Diagnostic et de Soutien à la Protection des Végétaux,

Direction de la Production Végétale (DPV), Bénin, Porto-Novo

Ecole d'Horticulture et d'Aménagement des Espaces Verts (EHAEV),

Université d’Agriculture de Kétou, Bénin, Kétou

\section{Affokpon A.}

Centre de Recherches Agricoles Sud-Bénin, Institut National des Recherches Agricoles du Bénin, Bénin, Allada

Département de Production Végétale, Faculté des Sciences Agronomiques, Université d’Abomey-Calavi, Bénin, Cotonou

\section{Bokonon-Ganta A. $\mathrm{H}$.}

Laboratoire de Diagnostic et de Soutien à la Protection des Végétaux,

Direction de la Production Végétale (DPV), Bénin, Porto-Novo

Département de Production Végétale, Faculté des Sciences Agronomiques,

Université d’Abomey-Calavi, Bénin, Abomey-calavi

\section{doi: 10.19044/esj.2016.v12n33p350 URL:http://dx.doi.org/10.19044/esj.2016.v12n33p350}

\begin{abstract}
Pineapple [Ananas comosus (L.) Merril], cultivated in Benin for it economical importance is affected by Pineapple mealybug wilt, a viral disease. The objectives of this study were to evaluate the determining factors of its prevalence and farmers knowledge on the disease. To achieve these, a total number of 180 farmers, distributed in five communes of southern Benin have been interviewed in 2014 and 2015. In order to identify factors that influence the disease distribution, generalized linear models with the binomial and multinomial distribution family were used to explain the effect of the variables (field and plant levels) and disease severity, respectively. Only factors 'commune' 'and' year ' had a significant effect on the incidence
\end{abstract}


at field level (69\% in 2014 and 78\% in 2015) while three predictors (township, year and variety) explained the severity of the disease and the interaction year -township was significant. Farmers perceive the disease and attribute five causes responsible for its expression. They use height methods to manage the disease. These findings constitute a qualitative as well as quantitative information to build on in designing methods of controlling the disease in order to boost the productivity of pineapple in Benin.

Keywords: Distribution, farmers’ perception, wilt, pineapple, Benin

\section{Resume}

L’ananas [Ananas comosus (L.) Merril], cultivé au Bénin pour son importance économique, est essentiellement confronté au virus communément appelé la maladie du wilt. L’évaluation des facteurs influençant sa prévalence et les connaissances des producteurs relatives à la manifestation de la maladie est la préoccupation majeure qui justifie la présente étude. A cet effet, cinq communes ont été prospectées au sud-Bénin au cours des années 2014 et 2015 où 180 producteurs ont été enquêtés. En vue de l'identification des facteurs qui influencent sa distribution, des modèles linéaires généralisés avec la distribution de la famille binomiale et multinomiale ont été utilisés expliquant respectivement les variables incidence (niveaux champ et plant) et sévérité de la maladie. Seuls les facteurs ‘commune' et 'année' ont eu un effet significatif sur l'incidence des champs (69\% en 2014 et $78 \%$ en 2015) tandis que trois prédicteurs (commune, année et variété) ont pu expliquer la sévérité de la maladie et l'interaction année-commune s'est révélée significative. Les producteurs identifient bien la maladie et évoquent cinq causes responsables de sa manifestation. Ils utilisent huit méthodes pour la gestion de cette maladie. Ces informations constituent donc une base capitalisable aussi bien quantitative que qualitative dans l'élaboration des mesures de gestion pouvant être initiées contre la maladie du wilt afin d'améliorer la production de l’ananas au Bénin.

Mots clés : Distribution, perception des producteurs, wilt, ananas, Bénin

\section{Introduction}

L’ananas [Ananas comosus (L.) Merril] est une plante pérenne originaire de l'Amérique du sud (Ploetz, 2001). Il constitue le deuxième fruit tropical le plus exporté entre les pays du monde (FAO, 2012). Au Bénin, sa culture en pleine expansion est principalement destinée aux marchés locaux, régionaux et internationaux (Fassinou Hotegni et al., 2014). Avec un rendement moyen annuel estimé à 5341,1 $\mathrm{Kg} \mathrm{Ha}^{-1}$ à l'échelle nationale en 
2013 (FAO, 2015), l'ananas est un fruit produit principalement au sud-Bénin dans le département de l'atlantique qui détient à lui seule plus de $95 \%$ de la production nationale avec les communes de Zè, Abomey-calavi, Allada, Toffo et Tori qui sont les cinq zones de fortes production (Arouna et Amassè, 2005; Gbenou et al., 2006). En effet, les conditions pédoclimatiques du sud-Bénin sont propices à la culture de l'ananas et lui confèrent de bonnes qualités organoleptiques qui font détenir à l'ananas béninois un label prisé sur le marché (AMEN, 2012). Cependant la culture est sujette à certaines maladies et ravageurs à travers le monde, lesquels compromettent son rendement en fruit. La maladie du dépérissement de l'ananas, communément appelée «wilt» de l'ananas [Mealybug Wilt of Pineapple (MWP)], est l'affection virale, la plus répandue et la plus dévastatrice connue de l'ananas (Sether et al., 2001; Borroto et al., 1998). Elle a été décrite pour la première fois aux îles Hawaï en 1910 (German et al., 1992) où elle a entraîné des pertes de rendement de fruits variant entre 30 et $35 \%$ en fonction de l'âge de la plante au moment de l'apparition des symptômes de la maladie (Sether et Hu, 2002a). Cette dernière est causée par un complexe de virus appelé le «Pineapple mealybug wilt-associted virus » (PMWaV) transmis par deux espèces de cochenille, Dysmicoccus brevipes et Dysmicoccusneo brevipes (Sether et al., 2001, Shelter et al., 2002b). Elle se manifeste par une coloration rouge des feuilles qui, progressivement, perdent leur turgescence avec de sévère dessèchement au niveau de leur partie supérieure, suivi d'un flétrissement rapide de la plante (Gary et al., 2003). Les symptômes les plus évolués montrent le nanisme des plants ainsi que leur pourriture.

Des chercheurs se sont intéressés de très près à la gestion de la maladie. Aux îles Hawaï, il est démontré qu'elle se manifeste par la présence sur la plante de la cochenille et des symptômes de virus. Ce qui suppose que l'élimination de l'un ou l'autre inhiberait l'expression de la maladie (Hu et al., 1996; Hughes et Samita, 1998). Le contrôle de la cochenille peut se faire à l'aide d'insecticides ou d'ennemis naturels. Ce dernier semble induire des effets partiels dans la mesure où les cochenilles sont protégées par les fourmis (González-Hernández, 1999b). Ainsi, l’alternative serait de contrôler la population des fourmis et les résultats concluants ont été rapportés (González-Hernández, 1999a; Jahn et Beardsley, 2000). Il est aussi conseillé d'utiliser des méthodes culturales (Fournier et al., 2015) en incluant l'espacement des plants lors des semis. La production de plants sains en culture in vitro permet également de contrôler la maladie.

La gestion d'une maladie ne peut se faire sans l'action participative des producteurs qui sont les premiers acteurs de la chaîne. C'est ce sur quoi l'Institut de Recherche et d'Application des Méthodes du développement (IRAM, 1998) a mis l'accent en montrant que l'implication des producteurs 
aux programmes de développement des méthodes de lutte est la clé de succès de gestion d'une maladie.

Au Bénin, la maladie du wilt de l'ananas est annoncée, pour la première fois en 2008, par Fanou et Adikan. Il n'en demeure pas moins que sa manifestation est encore mal connue dans les zones de production. Depuis lors, aucune étude n’est disponible pour expliquer le fléau. Spécifiquement (i) les facteurs influençant la prévalence, (ii) la perception des producteurs et les (iii) méthodes de lutte utilisées pour la gestion de la maladie du wilt étant inconnues, il apparaît opportun d'envisager la présente étude, à travers un état des lieux du MWP au Sud-Bénin afin de parvenir à l'élaboration de nouvelles méthodes de lutte plus efficaces.

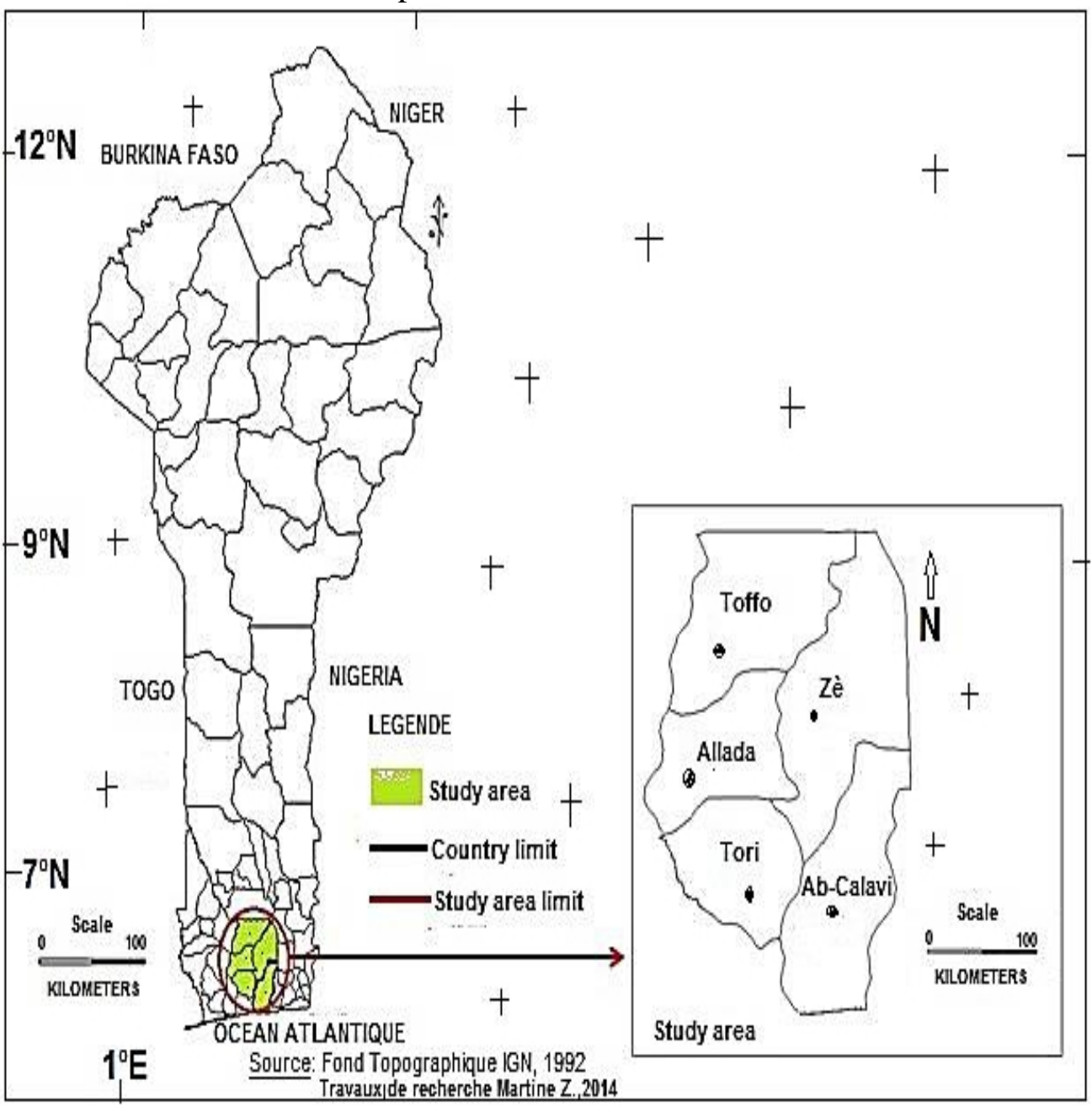

Figure 1: Carte des zones de prospection de la maladie du wilt 


\section{Matériel et Méthodes}

Milieu d'étude

L’étude a été conduite dans le département de l’Atlantique, au SudBénin. C'est une région humide qui compte deux saisons pluvieuses et deux saisons sèches avec une pluviométrie annuelle moyenne variant entre 1100 $\mathrm{mm}$ et $1400 \mathrm{~mm}$. Les températures annuelles moyennes oscillent entre $26^{\circ} \mathrm{C}$ et $28^{\circ} \mathrm{C}$ (Akoegninou et al., 2006). Elle est située dans la zone phytogéographique guinéo-congolaise caractérisée par des mosaïques de groupements végétaux sur sols sablonneux, des forêts semi-décidues et savanes arbustives sur des sols ferralitiques, hydromorphes et des vertisols. L’économie locale est basée sur la production de l'ananas, du maïs, du manioc et du palmier à huile. Les données ont été collectées dans les communes d'Abomey-Calavi, Allada, Toffo, Tori et Zè parce qu'elles constituent les zones de forte production d'ananas au Bénin (MAEP, 2012). Elles sont, de par leur positionnement naturel, réparties dans deux zones agro-écologiques dont la zone de terre de barre (Abomey-Calavi, Allada, Tori et Zè) et la zone de dépression (Toffo).

\section{Evaluation de l’incidence et de la sévérité de la maladie du wilt}

Des prospections effectuées pendant l'année 2014, de Septembre à Octobre, sont répétées en 2015 au cours de la même période. Les champs prospectés (tableau 1) sont distants de $5 \mathrm{Km}$, l'un de l'autre. La superficie des champs visités est d'au moins $800 \mathrm{~m}^{2}$ et des cultures d'ananas d'au moins 4 mois d'âge y sont érigées.

L’incidence et la sévérité sont évaluées sur 20 plants choisis au hasard dans chaque champ, suivant la méthode Zigzag (W) (Sether et al., 2010). L’incidence niveau champs et plant a été calculée à l'aide de l’équation proposée par Cooke (2006).

L’évaluation de la sévérité de la maladie du Wilt a été réalisée sur les mêmes plants selon l'échelle décrite par (Sether et al., 2001). Cette échelle comporte 4 niveaux: $0=$ pas de symptômes; $1=$ feuilles sèches perdant leur turgescence virant du rouge au rose; $2=$ flétrissement et distorsion des feuilles, nanisme de la plante; et $3=$ nanisme, pourriture et mort de la plante. La fréquence de chaque niveau de sévérité est calculée en fonction du champ selon la formule suivante (Banito et al., 2007):

$$
\mathrm{F}=\mathrm{Ni} / \mathrm{n}
$$

avec $\mathrm{F}=$ fréquence de la classe de sévérité dans le champ; $\mathrm{Ni}=$ nombre de plants affectés de la sévérité i; $n=$ nombre total de plants évalué.

Tableau 1 : Nombre de champs prospectés dans chaque commune par année

\begin{tabular}{ccccccc}
\hline & Abomey-Calavi & Allada & Tori & Zè & Toffo & Total \\
\hline 2014 & 11 & 34 & 13 & 13 & 21 & 92 \\
2015 & 20 & 26 & 20 & 20 & 20 & 106 \\
Total & 31 & 60 & 33 & 33 & 41 & 198 \\
\hline
\end{tabular}




\section{Evaluation des connaissances et de la perception des producteurs sur la maladie}

Une enquête a été conduite dans les cinq communes ciblées (Allada, Abomey-Calavi, Toffo, Tori et Zè) suivant trois critères principaux : i) les producteurs ayant au moins trois ans d'expérience dans la culture, ii) propriétaires de champs d'ananas et iii) la superficie de champs exploitée (au moins 0,5ha). Les enquêtes ont été réalisées par des interviews individuelles à l'aide des questionnaires semi-structurées. Les différentes rubriques du questionnaire sont relatives aux symptômes de la maladie du wilt de l'ananas, à ses causes, à la sensibilité des variétés, aux pertes enregistrées, aux différentes méthodes de lutte pour contrôler la maladie ainsi que leur efficacité. Les enquêtés ont été choisis au hasard dans chaque localité. Au total, 180 producteurs ont été retenus dans 57 villages dont 40 dans la commune d'Allada, 30 dans la commune d'Abomey-calavi, 31 dans la commune de Toffo, 39 dans la commune de Tori-Bossito et 40 dans la commune de Zè. La taille de l'échantillon (N) a été obtenue en utilisant l'approximation normale de la distribution binomiale proposée par Dagnelie (1998) :

$$
N=\left[\left(U_{1}-\alpha / 2\right)^{2} \times p(1-p)\right] / d^{2}
$$

Avec $\mathrm{P}$ (86,45\%), qui a été déterminée à partir de l'enquête exploratoire réalisée dans une localité d'étude par commune et représente la proportion des personnes qui remplissent les trois critères définis et $\mathrm{d}$ la marge d'erreur d'estimation, retenue à $5 \%$ dans cette étude. $U_{1}-\alpha /{ }_{2}=1,96$ et représente la valeur de la variable aléatoire normale pour un risque $\alpha$ égal à 0,05 . La marge d'erreur $d$ prévue pour tout paramètre à estimer à partir de l'enquête est de $5 \%$.

\section{Analyses statistiques}

Pour évaluer les facteurs influençant l'incidence au niveau des champs, un modèle linéaire généralisé avec la distribution de la famille binomiale est utilisé. Les facteurs testés sont la zone agro-écologique (terre de barre ou dépression), la commune (Abomey-Calavi, Allada, Toffo, Tori et Zè), l’année (2014 et 2015), la variété (Cayenne lisse et Pain de sucre), le mode de culture (culture associée ou non) et les adventices (présence ou absence). Le modèle complet incluant tous les facteurs et les interactions d’intérêt est spécifié. La fonction step AIC du package MASS (Venables et Ripley, 2002) du logiciel R version 3.2.1. (R Core Team, 2015) est utilisée en vue d'obtenir le modèle parcimonieux. Par simplification manuelle, la fonction ANOVA est utilisée pour compléter la démarche. Le pseudocoefficient de détermination R2 de Nagelkerke est calculé dans le package fmsb (Nakazawa ; 2012) pour déterminer le pouvoir explicatif du modèle obtenu. 
Les mêmes facteurs que précédemment sont utilisés pour tester l'incidence niveau plant. Le modèle linéaire généralisé à effets mixtes avec la distribution de la famille binomiale (le champ étant pris pour facteur aléatoire) a été utilisé dans le package lme4 (Bates et al., 2014). La simplification manuelle du modèle est obtenue en utilisant la fonction ANOVA (Zuur et al., 2009).

En ce qui concerne la sévérité, elle a été évaluée sur la base d’une échelle à quatre niveaux $0,1,2,3$. Ainsi, un modèle multinomial est utilisé pour identifier les facteurs affectant le niveau de sévérité. Cette analyse a été faite dans le package epicalc (Chongsuvivatwong, 2012). La simplification du modèle est aussi faite manuellement à l'aide de la fonction ANOVA. Les données d'enquête ont été soumises à la statistique descriptive (fréquence) et ont permis de présenter les résultats sous forme de graphes. Le test de khi2 a été utilisé pour comprendre si les proportions des différentes réponses des producteurs sont liées aux communes.

\section{Résultats}

\section{Incidence de la maladie du MWP}

\section{Incidence niveau champ}

Les facteurs 'commune' (ddl=4, déviance résiduelle $=192.33$, prob < 0.001 ) et 'année’ (ddl=1, déviance résiduelle $=185.19$, prob $=0.008$ ) ont un effet significatif sur l'incidence niveau champ. Le taux d'incidence est plus élevé en 2015 (78,37\%) (coef=0.584, prob =0.008) qu'en 2014 (68,53\%) (Figure 2). En ce qui concerne les communes, Allada présentait le taux d’incidence le plus élevé $(96,66 \%)$ (coef $=1.425$, prob $<0.001)$ alors que la commune de Tori présentait le taux d'incidence le plus faible (48,57\%) (coef=-0.656, prob = 0.043). Aucune différence significative n’a été observée entre Abomey-Calavi (70,96\%), Toffo (78,78\%) et Zè (60,97\%) $(\mathrm{P}>0,05)$ (tableau 2). Il ressort de ces résultats que l'incidence niveau champ varie en fonction du milieu et de la période de production.
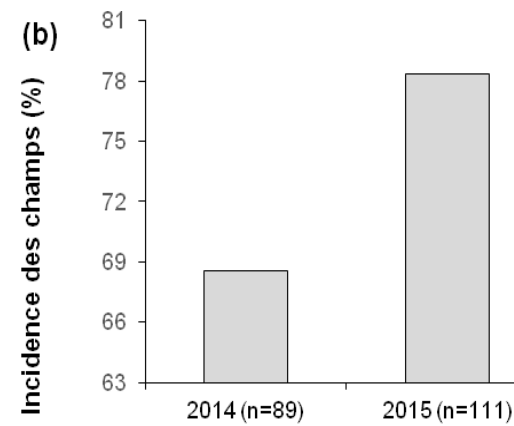

Années (a)

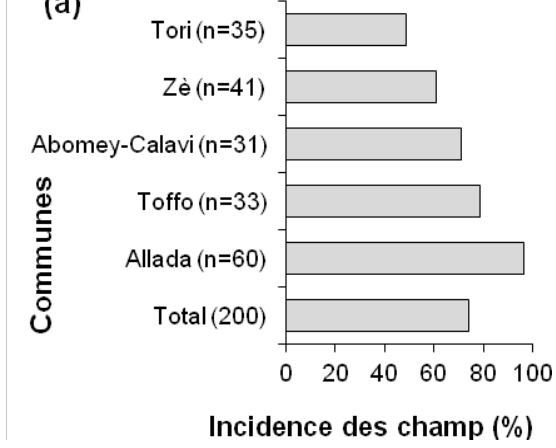

Figure 2 : Incidence niveau champs de la maladie du MWP de l'ananas au Sud-Bénin en 2014 et 2015 
Tableau 2 : Résultats du modèle linéaire généralisé avec la distribution de la famille binomiale : paramètres estimés du modèle

Tableau 2a : Résultat global des régressions logistiques binaires

\begin{tabular}{|c|c|c|c|c|c|c|}
\hline & ddl & $\mathrm{E}$ & ddl ( E) & $\mathrm{RE}$ & $\operatorname{Pr}(>$ Chi $)$ & Significativité \\
\hline NULL & 199 & 229,22 & & & & \\
\hline Commune & 4 & 36,89 & 195 & 192,33 & $<0,001$ & $* * *$ \\
\hline Année & 1 & 7,144 & 194 & 185,19 & 0,008 & $* *$ \\
\hline
\end{tabular}

Tableau $2 \mathbf{b}$ : résultat détaillé des régressions logistiques binaires

\begin{tabular}{cccccc}
\hline & Estimation & Ecart-type. & Erreur & Z-Valeur & Significativité \\
\hline (Intercept) & $-1176,26$ & 447,18 & $-2,63$ & 0,009 & $* *$ \\
CommuneAllada & 1,43 & 0,40 & 3,53 & $<0,001$ & $* * *$ \\
CommuneToffo & 0,28 & 0,35 & 0,80 & 0,426 & $\mathrm{~ns}$ \\
CommuneTori & $-0,66$ & 0,32 & $-2,02$ & 0,043 & $*$ \\
CommuneZè & $-0,19$ & 0,32 & $-0,60$ & 0,547 & $\mathrm{~ns}$ \\
Année2015 & 0,58 & 0,22 & 2,63 & 0,008 & $* *$ \\
\hline
\end{tabular}

Légende : non significatif 'ns' : P >0,05, '***' : P <0,001, '**' : P <0,01, '*': P <0,0 5

\section{Incidence niveau plant}

Trois facteurs se sont révélés déterminants dans l'incidence niveau plant : commune, année et variété. L’interaction entre commune et année était aussi significative indiquant que les différences entre années varient en fonction des communes et vice-versa (tableau 3). L'incidence était, en général, plus élevée en 2015 dans la commune d’Allada (19,04\%) suivie de Toffo $(17,50 \%)$ et de Zè $(15,50 \%)$ et d'Abomey-calavi $(14,25 \%)$, tandis que Tori a enregistré la plus faible valeur d'incidence (6,80\%) (figure 3 ). L'incidence était par contre faible pour la variété pain de sucre $(16,89 \%)$ (coef=-0.714, prob <0.001) comparée à la variété Cayenne lisse $(31,69 \%)$.

(a)
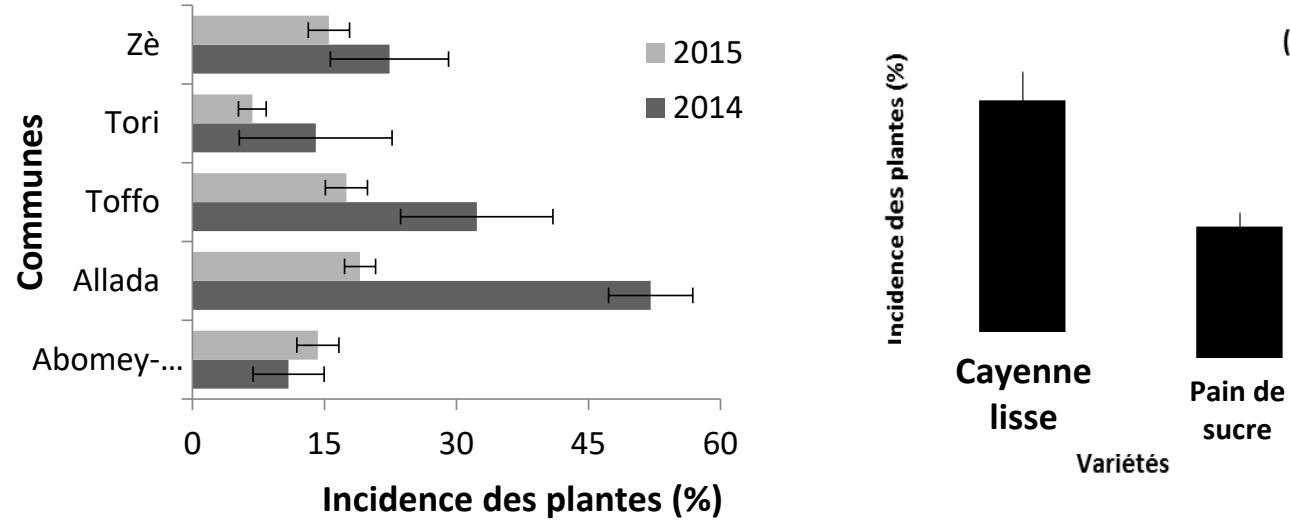

Figure 3 : (a) Incidence niveau plant (b) Incidence de la maladie du MWP de l'ananas au Sud-Bénin 
Tableau 3 : Résultats du modèle linéaire généralisé à effets mixtes avec la distribution de la famille binomiale (champ est le facteur aléatoire): paramètres estimés du modèle

Tableau 3a : résultat global des régressions logistiques binaires

\begin{tabular}{ccccccc}
\hline & ddl & E & Ddl & RE & $\operatorname{Pr}(>$ Chi $)$ & Significativité \\
\hline NULL & 3958,00 & 4209,70 & & & & \\
Commune & 4,00 & 273,72 & 3954,00 & 3936,00 & $<0,001$ & $* * *$ \\
Année & 1,00 & 116,13 & 3953,00 & 3819,90 & $<0,001$ & $* * *$ \\
Commune:Année & 4,00 & 55,51 & 3949,00 & 3764,40 & $<0,001$ & $* * *$ \\
\hline
\end{tabular}

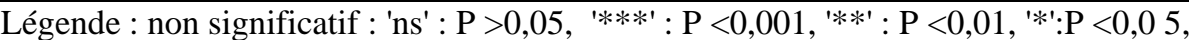
ddl=degré de liberté, $\mathrm{E}=$ écarts, $\mathrm{ddl}=$ degré de liberté, $\mathrm{RE}=$ Résidus des écarts, $\mathrm{Pr}=$ Probabiilité

Tableau $3 \mathbf{b}$ : résultat détaillé des régressions logistiques binaires

\begin{tabular}{cccccc}
\hline & Estimation & Ecart-type & Erreur & z-valueur & Significativité \\
\hline (Intercept) & $-2,51$ & 0,26 & $-9,56$ & $<0,001$ & $* * *$ \\
CommuneAllada & 2,59 & 0,24 & 10,62 & $<0,001$ & $* * *$ \\
CommuneToffo & 1,40 & 0,26 & 5,30 & 0,000 & $* * *$ \\
CommuneTori & 0,36 & 0,30 & 1,20 & 0,231 & $\mathrm{~ns}$ \\
CommuneZè & 0,96 & 0,25 & 3,79 & 0,000 & $* * *$ \\
Année2015 & 0,47 & 0,26 & 1,79 & 0,073 & $\mathrm{~ns}$ \\
CommuneAllada:Année2015 & $-2,30$ & 0,30 & $-7,57$ & 0,000 & $* * *$ \\
CommuneToffo:Année 2015 & $-1,14$ & 0,33 & $-3,48$ & 0,000 & $* * *$ \\
CommuneTori:Année2015 & $-1,26$ & 0,38 & $-3,30$ & 0,001 & $* * *$ \\
CommuneZè:Année2015 & $-0,86$ & 0,32 & $-2,66$ & 0,008 & $* *$ \\
\hline
\end{tabular}

Légende : non significatif : 'ns' : P >0,05, '***' : P <0,001, '**' : P <0,01, '*': $\mathrm{P}<0,05$

\section{Sévérité de la maladie du MWP}

Les facteurs déterminants du niveau de sévérité des attaques du wilt sont la commune, la variété et l'année. La figure 4 présente la proportion des niveaux de sévérité en fonction de ces facteurs déterminants. Ainsi, le risque pour un plant de souffrir de la sévérité de type 1 est 2, 78 fois plus grand dans la commune d'Allada qu'à Abomey-Calavi. Ce risque ne varie pas significativement pour les autres communes. Le risque pour un plant de souffrir de la sévérité de type 2 est 3,$08 ; 1,50$ et 1,36 fois plus grand respectivement dans les communes d'Allada, Toffo et Zè qu'à AbomeyCalavi. Ce risque est par contre 0,25 fois plus petit dans la commune de Tori. Le risque pour un plant de souffrir de la sévérité de type 3 est 3,04 et 2, 19 fois plus grand dans les communes d'Allada et Zè respectivement que dans la commune d'Abomey-Calavi. Ce risque est, par contre, 0,20 et 0,67 fois plus petit dans les communes de Toffo et Tori (tableau 4).

En général, le risque pour un plant de variété Pain de sucre de souffrir d'attaque de sévérité 1,2 et 3 est respectivement 0,37, 0,66 et 0,67 fois plus petit que celui d'un plant de variété Cayenne lisse, indiquant que les plants de variété Cayenne lisse sont exposés à une attaque plus sévère que les plants de variété Pain de sucre. 
Par ailleurs, le risque d'avoir un plant de sévérité niveau 3 était plus élevé en 2015 comparée à 2014. La tendance contraire est observée pour les deux autres niveaux de sévérité, pour lesquels, il y avait moins de risque en 2015 qu'en 2014.

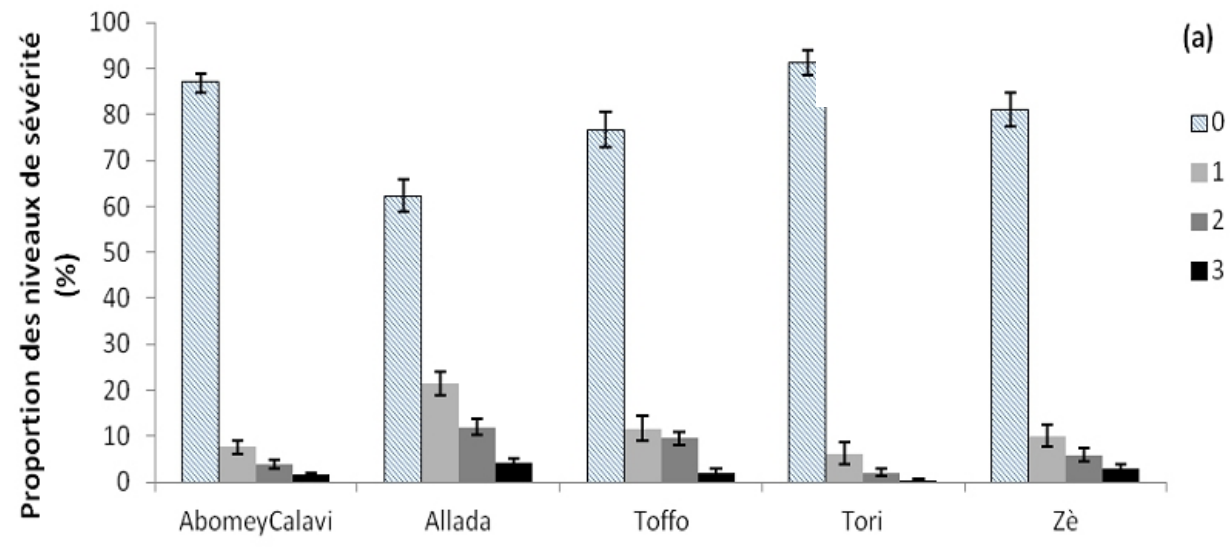

Communes

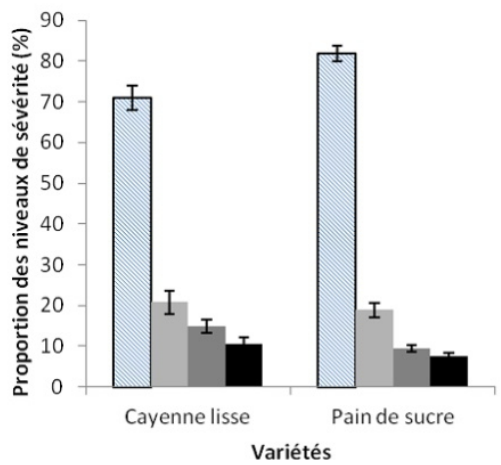

(b)

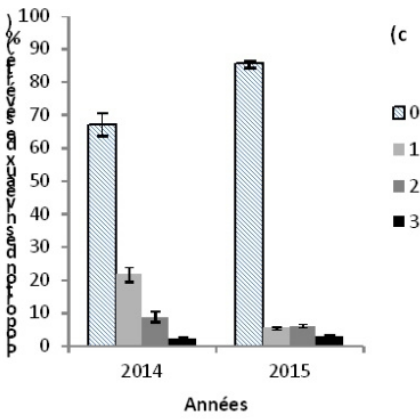

Figure 4 : Proportion des niveaux de sévérité de la maladie du MWP de l'ananas au Sud-Bénin

Tableau 4: Risque relatif de sévérité en fonction des facteurs retenus dans le modèle (niveau de référence = sévérité 0): risque relatif et erreur type.

\begin{tabular}{ccccc}
\hline & Coeff. & Erreur standard & RRS & 95\%IC \\
\hline (Intercept) & $2981,07^{* * *}$ & Sévérité 1 & & \\
CommuneAllada & $1,02^{* * *}$ & 0,00 & - & \\
CommuneToffo & 0,20 & 0,08 & 2,78 & $(2,36 ; 3,27)$ \\
CommuneTori & $-0,02$ & 0,12 & 1,22 & $(0,97 ; 1,54)$ \\
CommuneZè & 0,05 & 0,13 & 0,98 & $(0,76 ; 1,28)$ \\
Année2015 & $-1,48$ & 0,11 & 1,05 & $(0,85 ; 1,30)$ \\
& & 0,00 & 0,23 & $(0,23 ; 0,23)$ \\
\hline
\end{tabular}




\begin{tabular}{|c|c|c|c|c|}
\hline \multicolumn{5}{|c|}{ Sévérité 2} \\
\hline (Intercept) & $1121,02^{* * *}$ & 0,00 & - & \\
\hline CommuneAllada & $1,13 * * *$ & 0,10 & 3,08 & $(2,55 ; 3,72)$ \\
\hline CommuneToffo & $0,41 * * *$ & 0,10 & 1,50 & $(1,23 ; 1,84)$ \\
\hline CommuneTori & $-0,28 * * *$ & 0,04 & 0,75 & $(0,70 ; 0,81)$ \\
\hline CommuneZè & $0,31 *$ & 0,13 & 1,36 & $(1,06 ; 1,75)$ \\
\hline Année2015 & $-0,56$ & 0,00 & 0,57 & $(0,57 ; 0,57)$ \\
\hline \multicolumn{5}{|c|}{ Sévérité 3} \\
\hline (Intercept) & -346.04 & 0,00 & - & \\
\hline CommuneAllada & $1,11 * * *$ & 0,13 & 3,04 & $(2,38 ; 3,88)$ \\
\hline CommuneToffo & $-0,22 * * *$ & 0,01 & 0,80 & $(0,78 ; 0,82)$ \\
\hline CommuneTori & $-1,00$ & 0,01 & 0,37 & $(0,36 ; 0,37)$ \\
\hline CommuneZè & $0,79 * * *$ & 0,10 & 2,19 & $(1,81 ; 2,66)$ \\
\hline Année2015 & $0,17 * * *$ & 0,00 & 1,19 & $(1,19 ; 1,19)$ \\
\hline
\end{tabular}

\section{Connaissances des producteurs quant à la maladie du MWP}

Tous les producteurs enquêtés connaissent et identifient bien la maladie du wilt de l'ananas. Ils la reconnaissent facilement par la coloration rouge et la turgescence des feuilles (99,44\%), de même que le flétrissement et la distorsion (53,88\%). Cependant, $1,11 \%$ reconnaissent le nanisme et la pourriture des plantes. C'est dans les communes d'Allada et de Zè que ce niveau de sévérité de la maladie a été reconnu par les producteurs (2,5\%). Ils ont enregistré des pertes importantes dues à la maladie du wilt. Un pourcentage de $27,78 \%$ des producteurs disent avoir enregistré plus de $50 \%$ de pertes contre 3,33\% n'ayant enregistré aucune. Ces pertes sont relatives à la sensibilité de la variété d'ananas produite. Plus de la moyenne des producteurs enquêtés pensent que la variété Cayenne lisse est plus vulnérable à la maladie (61,66 \%). Cette idée est partagée par la majorité des producteurs dans toutes les communes sauf à Tori $(17,94 \%)$ où la plupart des producteurs pensent que la variété Pain de sucre apparaît plus vulnérable (Tableau 5). Un faible nombre de producteurs $(27,27 \%)$ enquêtés affirment que la variété Pain de sucre est plus sensible pendant que 6,68\% estiment que la pression de la maladie est la même à l'égard des deux variétés. Seuls, 3,54\% n’ont aucune idée de la variété la plus sensible.

\section{Perception des producteurs sur les causes de la maladie du wilt de l'ananas}

Au total, cinq causes selon les producteurs enquêtés sont à l'origine de la maladie du wilt de l'ananas au Bénin. Il s'agit notamment de: la cochenille de l'ananas, l'infertilité du sol, l'absence d'apport de fertilisant, la dégénérescence variétale, le mauvais labour. La cochenille de l'ananas est bien reconnue par les producteurs $(22,22 \%)$ comme vecteur de la maladie. Selon 13,25\% des producteurs enquêtés, lorsque le sol est surexploité et 
appauvri en nutriments, la maladie gagne facilement les plantes. Certains évoquent l'absence d'apport de fertilisants en temps réel aux plantes. L'absence d'apports de fertilisants évoquées par 2,22\% des producteurs est une cause due à un manque de moyen financier. Mais les producteurs de Tori et de Zè n’ont pas listé l'apport des fertilisants et le labour comme facteur limitant.

La dégénérescence variétale est également évoquée, mais uniquement dans la commune de Toffo (3,22\%). Le mauvais labour est évoqué par $2,22 \%$ des producteurs comme une cause de la maladie du wilt. Pour ces producteurs, le labour et le nivellement sont des phases importantes assurant un bon développement aux plantes qui arrivent ainsi à résister au wilt. Un taux de 69,44\% des producteurs enquêtés n'ont aucune connaissance des causes du wilt. Il faut souligner que dans les cinq communes, la cochenille est la cause la plus citée des producteurs.

\section{Les méthodes de lutte adoptées par les producteurs et leur efficacité pour le contrôle de la maladie de MWP}

$\mathrm{Au}$ total, huit méthodes de lutte ont été recensées auprès des producteurs $(72,78 \%)$ dans la gestion de la maladie du wilt. Seulement 27,22\% des producteurs enquêtés n'utilisent aucune méthode de lutte contre $72,78 \%$ qui pratiquent au moins une méthode de lutte.

\section{$\checkmark \quad$ Destruction des plantes malades}

La plupart des producteurs (58,33\%) utilise la méthode de destruction des plants malades par arrachage complet à la main ou à la houe et leur remplacement par de nouveaux rejets. Cette méthode est mise en œuvre, les quatre premiers mois de la croissance des plantes et représente pour les producteurs la méthode la plus pratiquée. Cependant, certains pensent qu'elle est loin d'être efficace pendant que d'autres (40,95\%) estiment qu'elle l'est peu.

\section{$\checkmark \quad$ Apport de fertilisants}

Pour ce qui est de l'apport d'engrais, 4,44\% des producteurs pensent que l'apport des fertilisants (Azote, Potassium, Phosphore et Magnésium) en temps réel (4, 8 et 11 mois) peut permettre aux plantes de résister à la maladie du wilt. $75 \%$ des producteurs ont témoigné de l'efficacité des apports au moment où certains (12,5\%) doute de son efficacité et le reste (12,5\%) pensent que l'apport n'a aucun effet sur la maladie. Il est à noter que les producteurs de Zè ne font aucun apport.

\section{$\checkmark \quad$ Sélection des rejets sains}

La sélection des rejets sains permet d'éviter l'apparition précoce de la maladie dans le champ. Seuls les producteurs des communes d'AbomeyCalavi (3,33\%) et de Toffo (6,45\%) adoptent cette technique pour prévenir la maladie. Parmi eux, certains pensent qu'elle est efficace (66.67\%) et le reste 33,33\% estiment qu'elle ne l'est pas. 


\section{$\checkmark \quad$ Contrôle de la cochenille de l’ananas (Dysmicoccus brevipes)}

Un pourcentage de $6,67 \%$ pense que le contrôle de la cochenille de l'ananas pourrait limiter la propagation de la maladie de MWP. Cette méthode est pratiquée dans toutes les communes enquêtées sauf dans la commune de Tori. Une proportion de 33,33\% des producteurs ont trouvé qu'elle est efficace contre $16,66 \%$ qui ont pensé qu'elle est peu efficace. Ceux qui pensent que cette méthode n'est pas efficace font un pourcentage de $50 \%$.

\section{$\checkmark \quad$ Couper les feuilles des plantes malades}

Une proportion de $2,77 \%$ des producteurs enquêtés appliquent cette méthode. Elle est particulièrement pratiquée par les producteurs de la commune de Toffo (12,9\%) et de Zè (2,5\%). Parmi eux, 80\% ont témoigné de son efficacité contre $20 \%$ qui ont trouvé qu'elle ne l'est pas.

\section{$\checkmark \quad$ Un bon labour et une bonne plantation}

Un pourcentage de 2,77\% des producteurs a mentionné l'utilité de la méthode et leurs avis ont été enregistrés dans les communes d'AbomeyCalavi (3,33\%), Toffo (9,68\%) et Tori (2,56\%). Elle est appréciée par 60\% des producteurs qui pensent qu'elle est efficace contre $20 \%$ qui disent qu'elle est peu efficace. Seuls, 20\% témoignent de son inefficacité.

\section{$\checkmark \quad$ La jachère}

Un pourcentage de 1,11\% des producteurs applique la jachère dans les communes d'Allada (2,5\%) et de Toffo (3,22\%), 50\% de ces producteurs pensent qu'elle est efficace tandis que les $50 \%$ restant pensent le contraire.

\section{$\checkmark \quad$ L'apport de fientes de volailles}

L'apport de fientes de volailles permet de relever le niveau de fertilité du sol. Elle est appliquée par 1,11\% des producteurs enquêtés dans les communes de Toffo (3,23\%) et de Tori (2,56\%). Parmi eux, 50\% témoignent qu'elle est efficace dans la gestion de la maladie du wilt contre $50 \%$ qui la jugent d'inefficace.

Tableau 5 : Perception des producteurs dans les différentes communes

\begin{tabular}{cccccc}
\hline \multicolumn{7}{c}{ Proportions des producteurs (\%) } \\
\hline Variables & $\begin{array}{c}\text { Abomey- } \\
\text { Calavi }\end{array}$ & Allada & Toffo & Tori & Zè \\
\hline \multicolumn{7}{c}{ Symptômes de la maladie du wilt * } \\
\hline Symptôme 1 & 100 & 100 & 100 & 100 & 97,5 \\
Symptôme 2 & 33,33 & 22,5 & 93,54 & 61,53 & 62,5 \\
Symptôme 3 & 0 & 2,5 & 0 & 0 & 2,5 \\
\hline \multicolumn{7}{c}{ Pertes de rendement*** } \\
\hline 5-9\% & 3,33 & 5 & 0 & 2,56 & 5 \\
$10-24 \%$ & 20 & 7,5 & 6,45 & 23,07 & 10 \\
$25-49 \%$ & 26,66 & 27,5 & 16,12 & 51,28 & 17,5 \\
$50 \%$ et plus & 33,33 & 25 & 48,38 & 17,5 & 17,5 \\
\hline \multicolumn{7}{c}{ Vulnérabilité des variétés*** } & 5,12 & 50 \\
\hline Aucune & 0 & 2,5 & 0 & 7,69 & 7,5 \\
connaissance & 40 & 5 & 0 & 71,79 & 17,5
\end{tabular}




\begin{tabular}{|c|c|c|c|c|c|}
\hline Cayenne lisse & 56,66 & 75 & 100 & 17,94 & 65 \\
\hline $\begin{array}{l}\text { Les deux } \\
\text { variétés }\end{array}$ & 3,33 & 17,5 & 0 & 2,5 & 10 \\
\hline \multicolumn{6}{|c|}{ Causes de la maladie du wilt } \\
\hline $\begin{array}{c}\text { Aucune } \\
\text { connaissance }\end{array}$ & 73,33 & 77,5 & 58,06 & 69,23 & 67,5 \\
\hline Cochenille & 16,67 & 15 & 38,71 & 17,94 & 25 \\
\hline Infertilité du sol & 13,33 & 10 & 12,9 & 15 & 15 \\
\hline $\begin{array}{l}\text { Absence } \\
\text { d'apport de } \\
\text { fertilisants }\end{array}$ & 3,33 & 5 & 3,22 & 0 & 0 \\
\hline $\begin{array}{l}\text { Dégénérescence } \\
\text { variétale }\end{array}$ & 0 & 0 & 3,22 & 0 & 0 \\
\hline Mauvais labour & 3,33 & 0 & 6,45 & 0 & 2,5 \\
\hline \multicolumn{6}{|c|}{${ }^{b}$ Méthodes de lutte } \\
\hline $\begin{array}{c}\text { Aucune } \\
\text { connaissance }\end{array}$ & 3,33 & 40 & 6,45 & 35,89 & 32,5 \\
\hline DestrucP & 66,67 & 50 & 70,96 & 51,28 & 57,5 \\
\hline ApportF & 3,33 & 5 & 6,45 & 7,69 & 0 \\
\hline SelectRejets & 3,33 & 0 & 6,45 & 0 & 0 \\
\hline ControlCA & 3,33 & 5 & 19,35 & 0 & 7,5 \\
\hline CpFeuilles & 0 & 0 & 12,9 & 0 & 2,5 \\
\hline LabPlant & 6,67 & 0 & 9,68 & 2,56 & 0 \\
\hline Jarch & 0 & 2,5 & 3,22 & 0 & 0 \\
\hline Fientes & 0 & 0 & 3,23 & 2,56 & 0 \\
\hline
\end{tabular}

symptôme 1: coloration rouge et turgescence des feuilles; symptôme 2: Flétrissement et distorsion des

feuilles; symptôme 3: pourriture et nanisme des plantes (b) DestrucPl= destruction des plantes;

ApportF $=$ apport de fertilisants ; SelectRejet $=$ sélection de rejets sains; $\mathbf{C o n t r o l C A}=$ contrôle de la

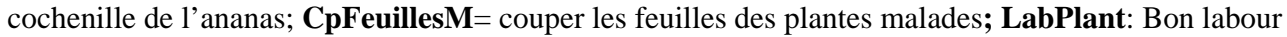
et une bonne plantation; Jarch= la jachère; FientesVol= apport de fientes de volailles.

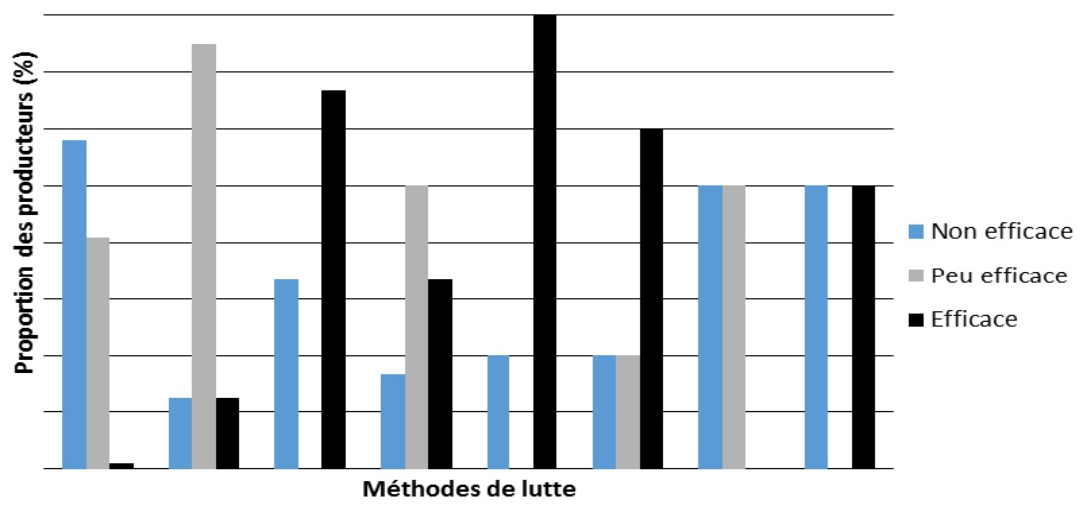

Figure 5 : Appréciation des producteurs sur les différentes méthodes de lutte dans la gestion de la maladie du wilt. 


\section{Discussion}

\section{Etat général de la maladie du wilt}

Plusieurs auteurs ont rapporté que la maladie de l'ananas la plus répandue au monde est la maladie du MWP (Gambley et al., 2008 ; Sether et al., 2005). La présente étude a montré que les facteurs: commune, année et variété ont un effet significatif sur la prévalence de la maladie du MWP au Bénin. La zone agro-écologique, la présence des adventices, et l'association de l'ananas à d'autres cultures n'ont pas d'effet significatif sur la prévalence de la maladie du MWP. Cependant, l'effet variété paraît raisonnable car, Carter et Collins (1947) ont montré que la variété Cayenne lisse est plus sensible à la maladie MWP. De même, selon l'étude effectuée au Bénin par Fanou et Adikan (2008), la sévérité de la maladie du wilt est liée à l'importance de la culture de la variété Cayenne lisse dans une localité. L'action anthropique des producteurs dans la gestion de la maladie et la méthode de culture justifient l'implication du facteur commune comme un prédicteur. Différentes méthodes de lutte sont utilisées par les producteurs pour le contrôle de la maladie. En exemple, l'utilisation des rejets sains pour la plantation, limitent l'apparition précoce de la maladie car, plus tôt le champ est infecté et plus vite son incidence s'accroit. Il y a également le contrôle de la cochenille (insecte vecteur) dont l'infestation des plants facilite l'apparition de la maladie. Ainsi, l'impact de la maladie du MWP sur le rendement peut être plus important sur des cultures qui sont infectées précocement durant leur cycle végétatif. Les producteurs qui parviennent à contrôler l'infestation de la cochenille peuvent donc influencer la manifestation de la maladie. A ce sujet, la présente étude révèle que certains producteurs assurent au mieux le contrôle de la cochenille.

Par ailleurs, nous avons constaté que la maladie s'est plus manifestée en 2014 qu'en 2015. Ce résultat pourrait s'expliquer par le fait que les rejets utilisés pour l'installation des champs au cours de la campagne agricole de 2015 sont moins infestés par les particules virales. Sether et al. (2001) ont montré que le taux d'infestation du virus PMWaV-2 dans les rejetscouronnes provenant des descendants est faible par rapport à ceux issus des plantes-mères.

\section{Connaissance des producteurs relativement à la maladie du wilt de l'ananas}

Les symptômes de la maladie tels que décrits par les producteurs correspondent bien à ceux mentionnées par la littérature scientifique. Ce qui traduit que ces producteurs ont une parfaite connaissance de la maladie. Le point de vue des producteurs quant à la variété la plus exposée à la maladie est diversifié. Ceux qui pensent que la variété Cayenne lisse est plus sensible confirment les résultats de Carter et Collins (1947) des îles Hawaï et Fanou 
et Adikan (2008) du Bénin. Les résultats sur l’incidence de la maladie du wilt de l'ananas obtenus au terme de la présente étude corroborent bien les pertes enregistrées par les producteurs.

La plupart des producteurs perçoivent la maladie. Cependant, très peu d'entre eux identifient son étiologie. En effet, les facteurs évoqués tels que l'infertilité du sol, le manque de fertilisant, la dégénérescence variétale et le mauvais labour n'expliquent pas l'étiologie de la maladie mais constituent des éléments de stress qui favorisent l'établissement de la maladie du wilt. Jahn et al. (2001) estiment que la sévérité de certaines maladies peut être modifiée par le renforcement de la fertilité du sol. Ce qui a justifié que certains producteurs pratiquent des techniques telles que l'apport de fertilisants, le bon labour, la jachère et l'apport de fientes de volailles pour freiner l'incidence de la maladie. La destruction des plantes malades est une méthode très ancienne. Mais la plupart des producteurs qui la pratiquent, pensent qu'elle n'est pas du tout efficace. Pour remédier à cette méthode certains producteurs proposent de couper les feuilles des plantes malades qui, selon eux, régénéreront de nouvelles feuilles saines. Ils pensent que cette méthode est très efficace. Toutefois, La sélection de rejets sains est une méthode préventive qui permettrait d'empêcher l'apparition précoce de la maladie de même que son augmentation.

\section{Conclusion}

La maladie du wilt est présente dans les principales communes de production de l'ananas au Bénin. Les prédicteurs commune et année expliquent sa prévalence au niveau des différents champs prospectés tandis que les prédicteurs commune et variété l'expliquent au niveau plants. Sa manifestation varie entre les différentes communes prospectées d'une année à une autre. Les facteurs, commune, variété et année sont ceux qui expliquent le mieux la sévérité de cette maladie. Tous les producteurs ont une bonne connaissance de la maladie mais très peu comprennent son étiologie. Ainsi donc, il faut remarquer que selon les producteurs la variété Cayenne est la plus vulnérable comparée à celle du pain de sucre. La présence de cochenille comme vectrice de la maladie, l'infertilité du sol, la surexploitation ou l'appauvrissement du sol et l'absence d'apport de fertilisants sont à l'origine de la maladie de wilt selon les producteurs. Certains parmi les producteurs d'ananas au Bénin s'investissent dans la lutte contre la maladie du wilt. Pour ce faire plusieurs méthodes sont utilisées dont les principales sont: la destruction des plants malades, le contrôle de la cochenille vectrice de la maladie, la coupure des feuilles des plants malades, l'apport de fertilisants et une bonne pratique culturale (labour et plantation). L'efficacité de ces différentes méthodes varie d'une commune à une autre. L'étiologie de la maladie du wilt de l'ananas doit donc être étudiée au Bénin 
afin que des mesures efficaces de lutte soient rapidement développées pour freiner la propagation de la maladie.

\section{Remerciements}

Nous remercions le Programme de Productivité Agricole en Afrique de l'Ouest (PPAAO) pour son appui financier dans la réalisation de cette étude. Nous tenons aussi à remercier Dr Kolawole Valère Salako pour sa contribution lors des analyses statistiques.

\section{References:}

1. Akoègninou, A. , Van der Burg, W. J. \& Van der Maesen, L. J. G. (2006). Flore Analytique du Bénin. Backhuys Publishers: Wageningen; 1034P.

2. AMEN (2012). Etude du prix plancher pour la filière ananas au Bénin, Rapport provisoire, Cotonou-BENIN, 44p

3. Arouna, A. \& Amassè, D. (2005). Analyse de la compétitivité de la filière ananas au Bénin. Cotonou : Institut National de Recherches Agricoles au Bénin (INRAB).

4. Banito, A., Verdier, V., Kpémoua, K. E \& Wydra, K. (2007). Assessment of major cassava diseases in Togo in relation to agronomic and environmental characteristics in a system approach. African Journal of Agricultural Research, 9, 418-428

5. Bates, D., Maechler, M., Bolker, B., \& Walker, S. (2014). lme4: Linear mixed-effects models using Eigen and S4. $R$ package version, 1(7).

6. Borroto EG, Cintra M, Gonzalez J, Borroto C, \& Oramas P. (1998). First report of closterovirus-like particle associted with pineapple plants (Ananas comosus cv. Smooth Cayenne) affected with pineapple mealybug wilt in Cuba. Plant Disease, 82 (2), 263-263.

7. Carter, W. (1945). Some etiological aspects of mealybug wilt. Phytopathology, 35, 305-315

8. Carter, W. \& Collins, J. L. (1947). Resistance to mealybug wilt of pineapple with special reference to a Cayenne-Queen hybrid. Phytopathology, 37, 332-348.

9. Cooke B.M. (2006). Disease assessment and yield loss. In: The epidemiology of plant diseases, Cooke B.M., Jones D.G. and Kaye B. (Eds.). 2nd Edn., Springer, Netherlands, ISBN: 10 1-4020- 4580-8, pp: 43-80.

10. Chongsuvivatwong, V., \& Chongsuvivatwong, M. V. (2012). Package 'epicalc'. Retrieved from CRAN: http://cran. rproject. org/web/packages/epicalc/epicalc. pdf. 
11. Dagnelie P. (1998). Statistique théorique et appliquée. Tome 2: Inférence statistique à une et à deux dimensions, De Boeck et Larcier, Paris et Bruxelles, p. 659.

12. Fanou, A. \& Adikan, B. (2008). Etude de l’importance et de la distribution des ravageurs et maladies d'ananas dans le département de l'Atlantique dans la perspective d'élaborer des méthodes de la lutte intégrée. In Promotion des Filières Agricoles Porteuses, Adjanohoun A, Igue K (eds). Actes de l'Atelier Scientifique National. Tenu à Abomey-Calavi, 19-22 Décembre 2006. Tome 1, 146-158.

13. FAO.(2015). Statistical databases.

Url : http://faostat3.fao.org/browse/Q/*/E Consulté le 15 Octobre, 2016

14. FAO. (2012).World ranking: Pineapples, by Production (tons). Url:http://mongabay.com/commodities/data/category/1-Production/1Crops/574-Pineaples/51-Production+(tonnes). Consulté le 24 Avril 2014.

15. Fassinou Hotegni, V. N., Lommen, W. J. M, van der Vorst, J. G. A. J., Agbossou, E. K. \& Struik, P. C. (2014). Bottlenecks and opportunities for quality improvement in fresh pineapple supply chains in Bénin. International Food and Agribusiness Management Review, 17(3) 139.

16. Fournier P., Soler A., Marie D. (2015). La culture de 1 'ananas victoria à la réunion pour l'exportation. Recueil de bonnes pratiques. CIRAD, France.

17. Gambley, C. F., Geering, A. D. W., Steele, V. \& Thomas, J. E. (2008). Identification of viral and non-viral reverse transcribing elements in pineapple (Ananas comosus) including members of two new badnavirus species. Arch.

18. Gary, C. J., John, W. B. \& González- Hermández, H. (2003). A Review of the Association of Ants with Mealybug Wilt Disease of Pineapple. Proc. Hawaiian Entomology. Society, 36, 9-28.

19. Gbenou, R. K., Taore, M. and Sissint, E. (2006). Etude accélérée de marché (EAM) sur les différents produist ananas au Bénin. Cotonou : Helvetas-Benin.

20. German, T. L., Ullman DE, Gunasinghe UB. 1992. Mealybugwilt of Pineapple. Advanced Disease Vector Research, 9, 241-259.

21. González-Hernández, H., Johnson, M. W. \& Reimer, N. J (1999a). Impact of Pheidolemegacephala (F.) (Hymenoptera: Formicidae) on the biological control of Dysmicoccusneobrevipes (Cockerell) (Homoptera: Pseudococcidae). BioControl, 15 (2), 145-152.

22. González-Hernández, H., Reimer, N. J. \& Johnson, M. W. (1999b). Survey of the natural enemies of Dysmicoccusmealybugs on pineapple in Hawaii. BioControl, 44, 47-58. 
23. Hu JS.,Sether DM, Ullman DE. (1996). Detection of pineapple closterovirus in pineapple plants and mealybugs, using monoclonal antibodies. Plant Pathology, 45, 829-836.

24. Hughes G, Samita S., 1998. Analysis of patterns of pineapple mealybug wilt disease in Sri Lanka. Plant disease, 82 (8), 885-890.

25. Jahn, G.C., Sanchez, E. R. \& Cox, P. G. (2001). The Quest for Connections: Developing a research agenda for integrated pest and nutrient management. IRRI Discussion Paper No. 42, International Rice Research Institute (IRRI), Los Baños (Philippines) 18 pp.

26. Jahn, G. C. \& Beardsley, J. W. (2000). Interactions of ants (Hymenoptera: Formicidae) and mealybugs (Homoptera: Pseudococcidae) on pineapple. Proc. Hawaii. Entomological Society. 34, 181-185.

27. MAEP (2012). Plan de développement à moyen terme de la filière Ananas dans les communes productrices de l'Atlantique. Ministère de l'Agriculture, de l'Elevage et de la Pêche, Cotonou, Bénin.

28. Nakazawa M. (2012). Package 'fmsb'. http://cran.rproject.org/web/packages/fmsb/fmsb.pdf

29. Ploetz, R.C. (2001). Significant diseases in the tropics that are caused by species of Fusarium. In: Summerell BA, Leslie JF, Backhouse D, Bryden WL, Burgees LW (eds), Fusarium: Paul Nelson Memerial Symposium. The American Phytopathological Society Press, St Paul, 295- 309.

30. Sether, D. M, Borth, W. B, Melzer, M. J \& and Hu, J. (2010). Spatial and temporal incidences of Pineapple mealybug wilt-associated viruses in pineapple planting blocks. Plant Disease, 94, 196-200.

31. Sether, D M., Melzer, M. J., Busto, J., Zee, F. \& Hu JS. (2005a). Diversity and mealybug transmissibility of ampeloviruses in pineapple. Plant Disease, 89, 450-456.

32. Sether, D. M., Melzer, M. J., Subere, C. V. \& Hu, J. S. (2005b). Pineapple mealybug wilt associated viruses I, 3, and 4, and Grapevine leafroll associated viruses 4, 5, 6, and 9 are a distinct group in the genus Ampelovirus. In Joint Meeting of the 3 Divisions of the International Union of Microbiological Societies; International Congress of Virology.

33. Sether, D. M \& Hu, J. S. (2002a). Yield impact and spread of Pineapple mealybug wilt-associated virus 2 and Mealybug wilt of pineapple in Hawaii. Plant disease, 86,867-874.

34. Sether, D. M. \& Hu, J. S. (2002b). Closterovirus infection and mealybug exposure are necessary for the development of mealybug wilt of pineapple disease. Phytopathology, 92, 928-935. 
35. Sether, D. M., Karasev, A. V., Okumura, C., Arakawa, C., Zee, F., Kislan, M. M., Busto, J. L., \& Hu, J. S. (2001). Differentiation, distribution, and elimination of two different Pineapple mealybug wilt-associted viruses Found in Pineapple. Plant Disease, 85, 856864.

36. Venables, W. N., \& Ripley, B. D. (2002). Random and mixed effects. In Modern Applied Statistics with S (pp. 271-300). Springer New York.

37. Zuur, A., Ieno, E. N., \& Meesters, E. (2009). A Beginner's Guide to $R$. Springer Science \& Business Media. 